\title{
Characterization of the pure-glue phase in QCD. Inclusion of the 2-body interactions thanks to the T-matrix formalism
}

\author{
Gwendolyn Lacroix ${ }^{* \dagger}$ Fabien Buisseret ${ }^{a}$ and Claude Semay \\ PNSN - Institut Complexys, \\ University of Mons - UMons, Place du Parc 20, 7000 Mons, Belgium. \\ ${ }^{a}$ Haute École Louvain en Hainaut, \\ HelHa, Chaussée de Binche 159, 7000 Mons, Belgium. \\ E-mail: gwendolyn.lacroix@umons.ac.be, claude.semay@umons.ac.be , \\ fabien.buissereteumons.ac.be
}

\section{Daniel Cabrera}

Departamento de Física Teórica II,

Universidad Complutense, 28040 Madrid, Spain.

E-mail: Daniel. Cabrera@fys.ucm.es

\begin{abstract}
The strongly coupled phase of Yang-Mills plasma with arbitrary gauge groups is studied thanks to a $T$-matrix approach with the input of lattice-QCD static potentials. The existence of 2-gluon glueballs (considered here as 2-adjoint bound states) as well as the equation of state computed within the Dashen, Ma and Bernstein's formalism are discussed. Results for $\mathrm{SU}(N)$ and $G_{2}$ gauge groups are obtained and compared to current lattice data and $G_{2}$ models.
\end{abstract}

Xth Quark Confinement and the Hadron Spectrum,

October 8-12, 2012

TUM Campus Garching, Munich, Germany

* Speaker.

${ }^{\dagger}$ G.L. and C.S. thanks the F.R.S-FNRS for financial support.

¥D.C. acknowledges financial support from Centro Nacional de Física de Partículas, Astropartículas y Nuclear (CPAN, Consolider - Ingenio 2010). 


\section{Introduction}

The thermodynamic features of Yang-Mills plasma with arbitrary gauge groups are studied by resorting to a $T$-matrix approach [1]. The equation of state $(\mathrm{EOS})$ is then computed thanks to the Dashen, Ma and Bernstein's formulation of statistical mechanics in terms of the $S$-matrix (or $T$-matrix) [2]. Such a formulation is particularly well suited for systems whose microscopic constituents behave according to relativistic quantum mechanics. Indeed, within the Yang-Mills plasma, gluons (adjoint particles) are seen as the effective degrees of freedom. Moreover, the use of a $T$-matrix formulation allows us to investigate the temperature range where the matter is strongly interacting. This strong interaction means here that glueballs are expected to still survive above $T_{c}$.

Altough the approach is formulated for any gauge group, the focus is on $\mathrm{SU}(N)$ and the large$N$ limit, and on $\mathrm{G}_{2}$ because comparisons with pure-gauge lattice data [3, 4] and $G_{2}$ models [5] can be drawn.

The proceeding is organized as follows. Sec. 2 is dedicated to the presentation of quasiparticle approach that we have used to describe the thermodynamic features of the Yang-Mills plasma. Note that a more general presentation of the quasiparticle approach including quarks is developed in [1]. The existence of bound states inside the gluon plama is discussed in Sec. 3 as well as the computation of the EoS. Finally, Sec. 4 is devoted to conclusions and perspectives.

\section{Quasiparticle approach for a Yang-Mills plasma}

Within our approach, the thermodynamic features of the Yang-Mills plasma are studied thanks to the use of the $T$-matrix and then, the grand canonical potential is computed by using the formalism presented in [2]. A summary of these two main steps is presented hereafter.

\subsection{T-matrix and Lippmann-Schwinger equation}

To obtain the $T$-matrix of the system, the Lippmann-Schwinger equation for the off-shell $T$ matrix $(\mathscr{T})$, schematically given by

$$
\mathscr{T}=V+V G_{0} \mathscr{T} \quad \text { with } G_{0} \text { the free propagator, }
$$

has to be solved for all the channels that we consider. A crucial ingredient of (2.1) is the interaction potential, $V$. In the present quasiparticle approach, we have made the choice to use a two-body potential directly provided by lattice QCD. Indeed, accurate computations of the quenched SU(3) static free energy of a quark-antiquark pair bound in a color singlet, $F_{1}(r, T)$, are available in [6].

Since we only consider the gluon plasma, we have to transpose $F_{1}(r, T)$ from a quark-antiquark pair to a gluon-gluon one. For that, we assume the Casimir scaling in order to extract the color dependence from the potential. Following the arguments proposed in [1] and by introducing quadratic Casimirs $\left(C_{2}\right), V$ has schematically the form

$$
V_{\mathscr{C}}=\kappa_{\mathscr{C}} v
$$


where $\mathscr{C}$ is the pair representation and

$$
\kappa_{\mathscr{C} ; R_{1} R_{2}}=\frac{C_{2}^{\mathscr{C}}-C_{2}^{R_{1}}-C_{2}^{R_{2}}}{2 C_{2}^{a d j}},
$$

where $C_{2}^{a d j}$ and $C_{2}^{R_{i}}$ are respectively the quadratic Casimirs of the adjoint representation and of the representation of the particle $i$. The real function $v$ is assumed not to depend on color factors anymore. The validity of this assumption has partially been checked in pure gauge SU(3) lattice calculations [7].

However, it still remains a debate on the fact that the proper potential term to use in phenomenological approaches is $F_{1}$ or the internal energy $U_{1}=F_{1}-T \partial_{T} F_{1}$. Since an entropic contribution is subtracted from $F_{1}$ in $U_{1}$, causing $U_{1}$ to be more attractive than $F_{1}$, eventually leading to larger dissociation temperatures for bound states in the deconfined medium, $U_{1}$ is thus used as potential term here. The explicit fitted expression of the internal energy $U_{1}$ used in this work can be found in the Appendix A of [1].

According to (2.2) and using $U_{1}(r, T)$ extracted from quenched SU(3) lattice data for a quarkantiquark pair, the potential between two quasigluons in the color channel $\mathscr{C}$ is then given by

$$
V(r, T)=\frac{\kappa_{\mathscr{C} ; g g}}{\kappa_{\bullet} ; q \bar{q}}\left[U_{1}(r, T)-U_{1}(\infty, T)\right]
$$

where the long-distance limit of the potential has to be normalized to zero in order to ensure the convergence of the scattering equation and to perform the Fourier transform of $V(r, T)$ by using the formula

$$
V\left(q, q^{\prime}, \theta_{q, q^{\prime}}\right)=4 \pi \int_{0}^{\infty} d r r V(r) \frac{\sin (Q r)}{Q}, \quad \text { where } \quad Q=\sqrt{q^{2}+q^{\prime 2}-2 q q^{\prime} \cos \theta_{q, q^{\prime}}},
$$

and where $\theta_{q, q^{\prime}}$ is the angle between the momenta $\vec{q}$ and $\vec{q}^{\prime}$.

Nevertheless, in the above formula, we have to pay attention to the fact that, since the gluons are particles of helicity 1 , the basis states that we have to use are two-gluon helicity states (see [8]). As we assume $V$ to be spin independent, only the orbital angular momentum content of the helicity states has to be taken into account. According to a standard integration, the $L$-wave part of potential (2.5) reads

$$
V_{L}\left(q, q^{\prime}\right)=2 \pi \int_{-1}^{1} d x P_{L}(x) V\left(q, q^{\prime}, x\right)
$$

where $P_{L}$ is the Legendre polynomial of order $L$ and $x=\cos \theta_{q, q^{\prime}}$. Our choice is to focus on the scalar, pseudoscalar and tensor $J^{P}$ channels since they are the ligthest ones and thus, the most likely to form bound states. The potentials for each helicity channel are respectively

$$
\begin{aligned}
& V_{0^{+}}\left(q, q^{\prime}\right)=\frac{2}{3} V_{0}\left(q, q^{\prime}\right)+\frac{1}{3} V_{2}\left(q, q^{\prime}\right), \\
& V_{0^{-}}\left(q, q^{\prime}\right)=V_{1}\left(q, q^{\prime}\right), \\
& V_{2^{+}}\left(q, q^{\prime}\right)=\frac{2}{5} V_{0}\left(q, q^{\prime}\right)+\frac{4}{7} V_{2}\left(q, q^{\prime}\right)+\frac{1}{35} V_{4}\left(q, q^{\prime}\right) .
\end{aligned}
$$

Once $V_{J^{P}}\left(q, q^{\prime}\right)$ is known, the off-shell $T$-matrix can be computed from (2.1) as follows in [9]:

$$
\mathscr{T}\left(E ; q, q^{\prime}\right)=V_{J^{P}}\left(q, q^{\prime}\right)+\frac{1}{8 \pi^{3}} \int_{0}^{\infty} d k k^{2} V_{J^{P}}(q, k) G_{0}(E ; k) \mathscr{T}\left(E ; k, q^{\prime}\right),
$$


where $E$ is the total energy in the center of mass frame and the two-gluon propagator reads

$$
G_{0}(E ; k)=\frac{m_{g}^{2}}{\varepsilon(k)} \frac{1}{E^{2} / 4-\varepsilon(k)^{2}-2 i \varepsilon(k) \Sigma_{I}}
$$

with the gluon dispersion relation $\varepsilon(k)=\sqrt{k^{2}+m_{g}^{2}}$. Note that the normalization conventions of the $T$-matrix are explained in details in [1]. The parameter $\Sigma_{I}$ accounts for the imaginary part of the gluon self-interaction, whereas the real part is reabsorbed in the effective gluon mass. In the present case, we approximate the small imaginary part for numerical purposes by $\Sigma_{I}=0.01 \mathrm{GeV}$ as in [9]. For the effective gluon mass, we follow the suggestion made in [10]: The nonzero value of $U_{1}(\infty, T)$ should eventually be responsible of an effective in-medium contribution to the gluon mass. The intuitive argument is that, when both gluons are infinitely separated, they no longer interact. Therefore, the remaining potential energy should be seen as a manifestation of self-energy effects induced by the surrounding medium. These effects are encoded in the model as a mass shift from the "bare" quasigluon mass, whose value has still to be fixed.

Since $U_{1}(\infty, T)=2 m_{q}(T)$, the adaptation to the gluon case must be done by extracting the correct color-dependence. From HTL computations [11], the self-energy color dependence is given by $C_{2}^{q} / C_{2}^{a d j}$ at the first order when it is added in the propagator as a mass term $\left(\mathrm{m}^{2}\right)$, that means here that

$$
\frac{U_{1}(\infty, T)}{2}=m_{q}(T)=\sqrt{\frac{C_{2}^{q}}{C_{2}^{a d j}}} \Delta(T) .
$$

So, $m_{q}(T)=2 \Delta(T) / 3$ in the $\mathrm{SU}(3)$ case where $\Delta(T)$ is considered as universal. The gluon thermal mass thus reads

$$
\delta(T)=\sqrt{\frac{C_{2}^{g}}{C_{2}^{a d j}}} \Delta(T)=\Delta(T),
$$

since $C_{2}^{g}=C_{2}^{a d j}$. So, $\delta(T)$ is gauge-group independent. The effective in-medium gluon mass is finally given in our approach as

$$
m_{g}(T)^{2}=m_{0}^{2}+\delta(T)^{2} .
$$

where the value $m_{0}$ is fitted to $0.7 \mathrm{GeV}$ in order to reproduce the zero-temperature glueball masses (see [1]). Moreover, $m_{0}$ is a typical value for the zero-momentum limit of the gluon propagator at zero temperature. All the contributions are quadratically added as it is the case when one is dealing with bosonic propagators. The gluon mass is thus gauge-group independent.

It is obvious that the problem of the gluon mass is far more complicated than the simple prescription (2.14), that has to be seen as valid in a first approximation only. Indeed, some evidences seem to show us that a more refined gluon mass should probably be momentum-dependent $[12,13]$.

The above discussion gives a more precise meaning to the term "quasigluons" used in this paper: It denotes transverse particles in the adjoint representation of $\mathrm{SU}(N)$ that gain an effective mass $m_{g}(T)$ given by (2.14) and interact through the potential (2.4).

Using all these prescriptions, we can finally compute $\mathscr{T}\left(E ; q, q^{\prime}\right)$. In practise, the HaftelTabakin algorithm is used to solve (2.10) [14] and the on-shell $T$-matrix is readily obtained as $\mathscr{T}\left(E ; q_{E}, q_{E}\right)$, with $q_{E}=\sqrt{E^{2} / 4-m_{g}^{2}}$. 


\subsection{Grand canonical potential}

According to [2] and by following the procedure presented in [1], the grand canonical potential for the Yang-Mill plasma with an arbitrary gauge group reads

$$
\begin{aligned}
& \Omega=2 \operatorname{dim} a d j \omega_{0}\left(m_{g}\right)+\sum_{\mathscr{C}} \sum_{J^{P}} \operatorname{dim} \mathscr{C}(2 J+1)\left\{\omega_{0}\left(M_{\mathscr{C}, J^{P}}\right)\right. \\
& \left.+\frac{1}{2 \pi^{2} \beta^{2}} \int_{2 m_{g}}^{\infty} d \varepsilon \varepsilon^{2} K_{2}(\beta \varepsilon) \operatorname{Tr}_{\mathscr{C}, J^{P}}\left[(\delta \operatorname{Re} \mathscr{T})^{\prime}-2 \pi\left((\delta \operatorname{Re} \mathscr{T})(\delta \operatorname{Im} \mathscr{T})^{\prime}-(\delta \operatorname{Im} \mathscr{T})(\delta \operatorname{Re} \mathscr{T})^{\prime}\right)\right]\right\} .
\end{aligned}
$$

This formula takes only into account the two-body interactions since they are expected to be the dominant scattering processes.

The first term of (2.15) is the free ideal quasigluon gas; $\operatorname{dim} a d j$ is the dimension of the adjoint representation and

$$
\omega_{0}(m)=\frac{1}{2 \pi^{2} \beta} \int_{0}^{\infty} d k k^{2} \ln \left(1-\mathrm{e}^{-\beta \sqrt{k^{2}+m^{2}}}\right)
$$

is the grand potential per degree of freedom associated to the gluon. The second and third parts of (2.15) respectively represent the glueball and scattering contributions to the thermodynamics. Glueballs are considered as new free species inside the plasma with mass $M_{\mathscr{C}, J^{P}}$. The glueball mass is the energy associated to a pole in the on-shell $T$-matrix $\mathscr{T}\left(E ; q_{E}, q_{E}\right)$. The equation (2.10) must be solved for all channels in which two gluons can interact in order to compute the summation in (2.15). These channels are labelled by the $J^{P}$ number and the color representation, $\mathscr{C} ; \operatorname{dim} \mathscr{C}$ is the dimension of color representation. In the remaining trace of (2.15), it is understood that the $T$-matrix has been computed in a given two-body channel with color $\mathscr{C}$ and quantum number $J^{P}$, and that the Dirac $\delta$ reads $\delta(\varepsilon-2 \varepsilon(q))$. Notice that $K_{2}(x)$ is the modified Bessel function of the second kind, that $\beta=1 / T$, and that the symbol "prime" is the derivative respective to the energy.

\section{Results}

This section is divided into two parts: The existence of the glueballs above $T_{c}$ and the computation of the EoS. We focus our study on the ligthest glueballs: The scalar, pseudoscalar and tensor ones, and on $\mathrm{SU}(N)$ and $\mathrm{G}_{2}$ cases since they are the most studied groups in the literature. Since we consider the two-gluon interactions, the color channels that we have to consider are simply given by the tensor product of the adjoint representation by itself.

For $\mathrm{SU}(N)$ case:

$$
\begin{array}{rlrl}
(1,0, \ldots, 0,1) \otimes & (1,0, \ldots, 0,1)= & \\
& \bullet & & \\
& \oplus(1,0, \ldots, 0,1)^{A} \oplus(2,0, \ldots, 0,2)^{S} & & N \geq 2 \\
& \oplus(1,0, \ldots, 0,1)^{S} \oplus(0,1,0, \ldots, 0,2)^{A} \oplus(2,0, \ldots, 1,0)^{A} & & N \geq 3 \\
& \oplus(0,1,0, \ldots, 0,1,0)^{S} & & N \geq 4 .
\end{array}
$$

For $\mathrm{G}_{2}$ case:

$$
(0,1) \otimes(0,1)=\bullet^{S} \oplus(0,1)^{A} \oplus(0,2)^{S} \oplus(2,0)^{S} \oplus(3,0)^{A}
$$


Table 1: Masses (in units of $\sqrt{\sigma}=0.42 \mathrm{GeV}$ ) of lowest-lying glueballs above $T_{c}\left(T_{c}=0.3 \mathrm{GeV}\right)$. A dash marks the temperature at which either the bound or resonant states is not detected anymore.

\begin{tabular}{|c|c|c|c|c|c|c|c|c|c|c|}
\hline \multicolumn{2}{|c|}{ Channel } & \multicolumn{3}{|c|}{ Singlet } & \multicolumn{3}{c|}{ Adjoint $^{s}$} & \multicolumn{3}{c|}{$(2,0)^{S}$} \\
\hline \multicolumn{2}{|c|}{ Group } & \multicolumn{3}{|c|}{ All } & \multicolumn{3}{c|}{ SU $(N \geq 3)$} & \multicolumn{3}{c|}{$G_{2}$} \\
\hline$T / T_{c}$ & $2 m_{g}$ & $0^{++}$ & $0^{-+}$ & $2^{++}$ & $0^{++}$ & $0^{-+}$ & $2^{++}$ & $0^{++}$ & $0^{-+}$ & $2^{++}$ \\
\hline 1.05 & 6.50 & 4.52 & 5.43 & 5.43 & 6.00 & 6.45 & 6.31 & 6.14 & - & 6.38 \\
& & 6.48 & & & & & & & & \\
1.10 & 5.24 & 4.57 & 5.21 & 5.00 & 5.14 & - & - & 5.21 & & - \\
1.15 & 4.71 & 4.43 & - & 4.67 & & & & - & & \\
1.20 & 4.43 & 4.33 & & - & & & & & & \\
1.25 & 4.26 & 4.24 & & & & & & & & \\
1.30 & 4.14 & - & & & & & & & & \\
1.35 & - & & & & & & & & & \\
\hline
\end{tabular}

The superscript $S / A$ denotes a symmetric/antisymmetric channel. The first/second/third line in the $\mathrm{SU}(N)$ case exists as soon as $N \geq 2 / 3 / 4$. The dimensions and color factors of the representations appearing in (3.1) and (3.2) can be found in [1]. Note that only the symmetric representations of (3.1) and (3.2) will be taken into account in what follows since the $J^{P}$ channels that we consider are symmetric and Pauli's principle has to be respected.

\subsection{Existence of glueballs above $T_{c}$}

The bound and resonant states appear as poles in the on-shell $T$-matrix as previously mentionned. The corresponding masses are given in Table 1 in the different considered color and $J^{P}$ channels. We can notice that the masses of the color-singlet are the same for $\mathrm{SU}(N)$ at all $N$ and $G_{2}$ since, $\kappa_{\mathscr{C}} ; \bullet=-1$ for all gauge groups in the color-singlet channel and the gluon mass is independent of $N$. It can be also observed that the color-singlet channel survives up to $1.3 T_{C}$ in the $0^{+}$case (the most attractive $J^{P}$ channel). In the other color channels, the melting is quicklier due to the less bounded potential. Indeed, $\kappa_{\mathscr{C}} \geq-1$ in the other attractive color channels.

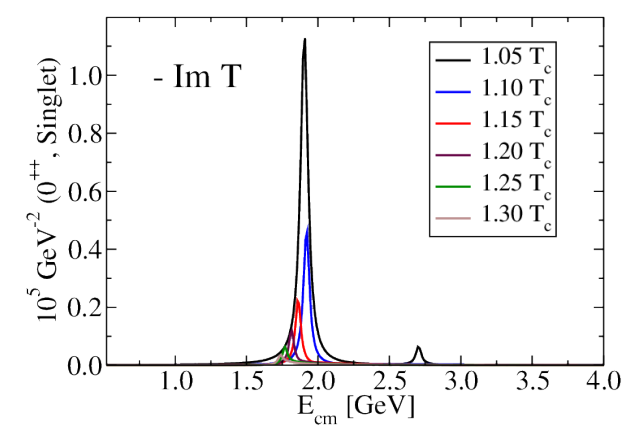

Figure 1: $\operatorname{Im} T$ for $g g$ scattering in the $0^{++}$singlet channel for various $T$ with $T_{c}=0.3 \mathrm{GeV}$. 
The evolution of the imaginary part of the on-shell $T$-matrix in the singlet scalar channel versus the temperature is displayed in Fig. 1: This gives an overall picture of the glueball progressive dissolution in the medium. The peak in the imaginary part, depicting a bound state, becomes broader and broader before melting into the continuum as the temperature is increased.

\subsection{Equation of state}

Using (2.15), the EoS can be computed. Then, the pressure is simply given by $p=-\Omega$. To establish more easily the comparison between our approach, lattice QCD and $G_{2}$ models, the pressure is normalized to the Stefan-Boltzmann pressure which is defined as

$$
p_{S B}=-2 \operatorname{dim} \operatorname{adj} \lim _{m \rightarrow 0} \omega_{0}(m)
$$

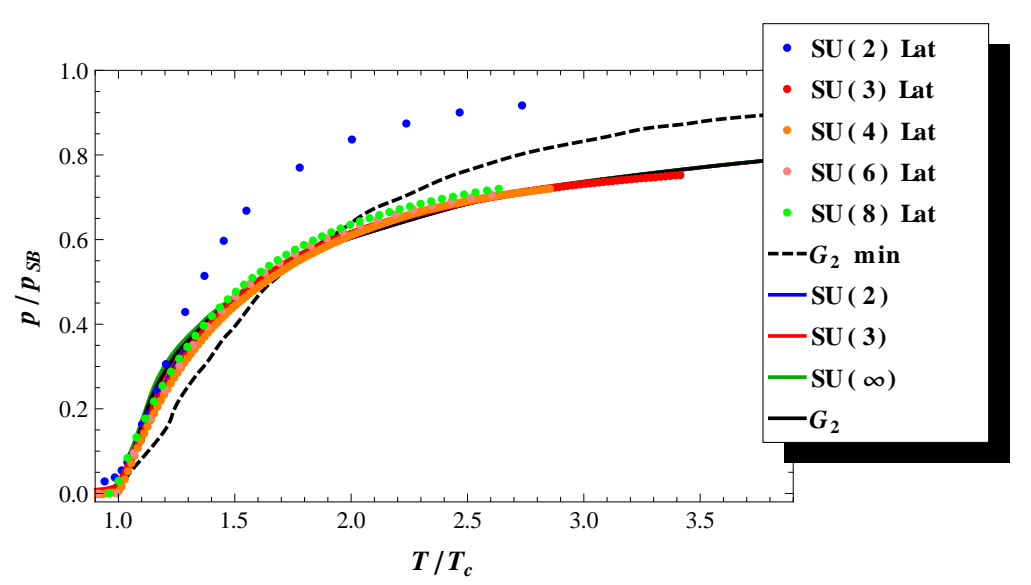

Figure 2: Normalized pressure versus temperature in units of $T_{c}$ (with $T_{c}=0.3 \mathrm{GeV}$ ), computed for the gauge groups $\mathrm{SU}(2,3, \infty)$ and $G_{2}$ (solid lines). Note that all the curves are nearly indistinguishable. Our results are compared to the lattice data of [3] for $\mathrm{SU}(2)$ (dots) and [4] for $\mathrm{SU}(3,4,6,8)$ (dots), and of the minimal $G_{2}$ model of [5] for $G_{2}$ (dashed line).

In Fig. 2, the normalized pressure are presented for different gauge groups: $S U(2), S U(3)$, $\mathrm{SU}(\infty)$ and $\mathrm{G}_{2}$. Severals remarks can be done. First, the free gluon thermodynamic contribution is gauge-group invariant once normalized to $p_{S B}$. The gauge-group dependence is only present in the bound state and scattering sectors. The number of allowed color channels (i.e. the symmetric ones) depends on the gauge group (see (3.1)) and determines the allowed maximum number of bound states and the number of scattering channels. The bound state thermodynamic contribution comes from two effets: The number and the mass of the existing glueballs. Because of the glueball dissociation, this contribution is only taken into account up to the temperature of dissociation (see Table 1). One can observe in Fig. 2 that the computed EoS are not very sensitive to the gaugegroup. The most important difference between the curves occurs between 1.05 and $1.35 T_{c}$ : In this range, the gluon-gluon interactions are maximal. When the temperature increases, the Born approximation becomes more and more valid and the pressure then scales as dim adj. Thus the normalized pressure tends to be universal. 
In Fig 2, it is also worth noticing that the EoS computed in our approach favorably compares with QCD lattice data for gauge groups SU(3-8) [4] where such universal curves seem to appear. For SU(2) [3], our results seems to underestimate the lattice computations. Nevertheless, it is important to notice that the available SU(2) lattice data are not recent and therefore, they are maybe not the most accurate and reliable ones. Concerning $\mathrm{G}_{2}$, no lattice data about EoS are currently available but a new effective matrix model describing pure Yang-Mills thermodynamics has been proposed in [5]. These last results are compared to ours in Fig 2.

\section{Conclusions}

The relevance of gluon-gluon interactions beyond the critical temperature in the pure gauge SU(3) plasma has been addressed in a non-perturbative $T$-matrix framework with the input of (Casimir-scaled) potentials from thermal lattice QCD and a model of quasigluon mass independent of the gauge group. Scalar glueball bound states in the singlet channel survive up to temperatures of about 1.3-1.5 $T_{c}$, together with sizable threshold effects due to strong correlations beyond the two-particle threshold. The EoS of the gluon-glueball gas is reproduced in good agreement with quenched lattice $\mathrm{SU}(N)$ simulations and with the predictions from $G_{2}$ models.

A natural extension to this paper is to study the light meson spectrum at finite temperature and the QCD EoS by including quarks within the model. Computations with baryonic potential can be also considered.

\section{References}

[1] G. Lacroix, F. Buisseret, C. Cabrera, and C. Semay [arXiv:1210.1716].

[2] R. Dashen, S.-K. Ma, and H. J. Bernstein, Phys. Rev. 187, 345 (1969).

[3] J. Engels, J. Fingberg, K. Redlich, H. Satz, and M. Weber, Z. Phys. C 42, 341 (1989).

[4] M. Panero, Phys. Rev. Lett. 103, 232001 (2009).

[5] A. Dumitru, Y. Guo, Y. Hidaka, C. P. K. Altes, and R. D. Pisarski [arXiv:1205.0137].

[6] O. Kaczmarek, F. Karsch, P. Petreczky, and F. Zantow, Phys. Lett. B 543, 41 (2002).

[7] S. Gupta, K. Huebner, and O. Kaczmarek, Phys. Rev. D 77, 034503 (2008).

[8] V. Mathieu, F. Buisseret, and C. Semay, Phys. Rev. D 77, 114022 (2008).

[9] D. Cabrera and R. Rapp, Phys. Rev. D 76, 114506 (2007).

[10] A. Mocsy and P. Petreczky, Phys. Rev. D 73, 074007 (2006).

[11] J. P. Blaizot, E. Iancu, and A. Rebhan, Phys. Lett. B 470, 181 (1999); Phys. Rev. D 63, 065003 (2001).

[12] A. Cucchieri and T. Mendes, Phys. Rev. D 81, 016005 (2010); O. Oliveira and P. Bicudo, J. Phys. G 38, 045003 (2011).

[13] J. M. Cornwall, Phys. Rev. D 26, 1453 (1982); D. Binosi and J. Papavassiliou, Phys. Rep. 479, 1 (2009); A. C. Aguilar, D. Binosi, J. Papavassiliou, and J. Rodriguez-Quintero, Phys. Rev. D 80, 085018 (2009); A. C. Aguilar, D. Binosi, and J. Papavassiliou, JHEP 1007, 002 (2010).

[14] M. I. Haftel and F. Tabakin, Nucl. Phys. A 158, 1 (1970). 